http://dx.doi.org/10.35381/racji.v5i3.1119

\title{
Proyecciones Laborales para las trabajadoras domésticas en el contexto del COVID-19
}

\section{Labour projections for domestic workers in the context of COVID-19}

\author{
Mercedes Carolina Navarro-Cejas \\ ur.mercedesnavarro@uniandes.edu.ec \\ Universidad Regional Autónoma de los Andes, Riobamba \\ Ecuador \\ https://orcid.org/0000-0003-4377-7250 \\ Lola Ximena Cangas-Oña \\ ur.lolacangas@uniandes.edu.ec \\ Universidad Regional Autónoma de los Andes, Riobamba \\ Ecuador \\ Jhoanna Nicole Bermeo-Alvarado \\ dr.jhoannamba90@uniandes.edu.ec \\ Universidad Regional Autónoma de los Andes, Riobamba \\ Ecuador \\ Cristian Patricio Naranjo-Moreno \\ dr.cristianpnm57@uniandes.edu.ec \\ Universidad Regional Autónoma de los Andes, Riobamba \\ Ecuador
}

Recibido: 31 de octubre de 2020

Revisado: 10 de octubre de 2020

Aprobado: 05 de diciembre de 2020

Publicado: 10 de diciembre de 2020 


\title{
RESUMEN
}

Dada la importancia del tema relacionado a la realidad de la pandemia, se presenta el siguiente trabajo cuyo objetivo principal de esta investigación es analizar las proyecciones laborales para las trabajadoras domésticas en el contexto del COVID19, en Ecuador y la vulneración de su trabajo. La investigación se desarrolló desde la perspectiva cuantitativa, de tipo documental, el diseño se circunscribe al bibliográfico. Dicho diseño utiliza procedimientos lógicos y mentales tales como: análisis, síntesis, deducción e inducción, mediante un análisis de contenido de normativas, leyes, informes, datos de organismo mundiales, relacionado al tema de estudio. Se concluye, que existe la necesidad de plantear alternativas con el fin proteger y tutelar los derechos de las trabajadoras domésticas, ya que la realidad que vendrá después de la pandemia seguramente será aún más complicada, por tal motivo, este sector de la sociedad debe contar con las garantías necesarias para continuar con la prestación de sus servicios.

Descriptores: Derecho al trabajo; pandemia; empleo de las mujeres. (Palabras tomadas del Tesauro UNESCO).

\begin{abstract}
Given the importance of the issue related to the reality of the pandemic, the following work is presented whose main objective of this research is to analyze the labor projections for domestic workers in the context of COVID-19, in Ecuador and the violation of their work. The research was developed from a quantitative perspective, documentary type, the design is limited to the bibliographic. This design uses logical and mental procedures such as: analysis, synthesis, deduction and induction, through an analysis of the content of regulations, laws, reports, data from world organizations, related to the subject of study. It is concluded that there is a need to propose alternatives in order to protect and protect the rights of domestic workers, since the reality that will come after the pandemic will surely be even more complicated, for this reason, this sector of society must count with the necessary guarantees to continue with the provision of its services.
\end{abstract}

Descriptors: Right to work; pandemic; employment of women. (Words taken from the UNESCO Thesaurus). 


\section{INTRODUCCIÓN}

Desde finales del año pasado se presentó en el mundo una crisis a nivel de todos los países producida por el virus SARS-CoV-2 y denominada oficialmente como COVID19. Las razones y el lugar en donde se originó la enfermedad no están claros científicamente, sin embargo, lo cierto es que todos los postulados doctrinales advierten de la alta facilidad con la que se propaga la enfermedad y los amplios aspectos negativos que abarca en todos los sectores sociales tanto económicos, laborales, de salud, entre otros.

Dentro de este contexto uno de los sectores afectados es sin duda el ámbito laboral el cual se ha visto modificado por las condiciones de la enfermedad que impiden, entre otras cosas, que se desarrollen actividades presenciales, por lo que ha existido una considerable afectación económica en todos los aspectos posibles. Sin embargo, la Constitución del Ecuador Ecuatoriana garantiza el trabajo como fuente de realización personal y base de la economía social a todos los ciudadanos sin discriminación alguna, el pleno respeto a su dignidad, una vida decorosa, remuneraciones justas, el desempeño de un trabajo saludable libremente escogido, y aceptado (Constitución de la República del Ecuador, 2008).

La falta de igualdad hacia la mujer y la vulneración de sus derechos primordiales provocan que no exista una tutela real de protección frente a la discriminación ocasionada, puesto que esta se puede presentar en varios ámbitos tales como el laboral, familiar, social, etc. Es por esta razón, que se debe procurar ejercer los derechos necesarios para que no se vean vulnerados por ningún motivo los principios de igualdad, ya que de esta manera se garantiza la posibilidad de estar libres de todo tipo de violencia, permitiendo a las mujeres tener la oportunidad de gozar una vida digna con igualdad de derechos. El derecho a la igualdad de trabajo de las mujeres tiene vital importancia, porque esta es la única manera de respetar los derechos humanos internacionales y en especial que deben ser cumplidos por todos los Estados que han firmado los mismos y entre ellos está el Ecuador, teniendo en cuenta que a las mujeres siempre se les ha tenido bajo la concepción que pertenecen a un grupo débil y que necesitan de la protección de las autoridades. (Sánchez y otros, 2019:41). 
De esta forma, en criterio de los autores de ésta investigación el tema sobre la discriminación puede ser muy amplio y de distintas clases, por lo que nos enfocaremos en la discriminación a la mujer doméstica. Desde tiempos inmemoriales la mujer ha sido maltratada y discriminada de diferentes formas, ya sea por su condición física, intelectual, laboral, social, etc. Debido a esto, cuando la mujer se dedica a las labores domésticas, se acentúa aún más el trato diferenciado que recibe por parte de sus superiores o familiares. Para Caamaño-Rojo (2010) se distinguen dos grades etapas en la legislación laboral para el reconocimiento de la mujer en este campo:

Si se atiende a los orígenes de la legislación laboral se pueden distinguir claramente dos grandes etapas en la evolución normativa protectora de la mujer trabajadora. En un primer momento, que en gran medida coincide con el paulatino perfilamiento del Derecho del Trabajo como un área normativa especial y autónoma, la tutela jurídica de las trabajadoras estaba orientada a la maternidad 40 y, además, a amparar su condición de mujer, acorde con una visión de la trabajadora como un sujeto de derecho más débil41 y, por lo tanto, en gran medida "necesitada" de una protección particular, similar en algunos casos a la que se le confería a los menores de edad (p.193).

En el marco de estas referencias sería importante considerar el informe de la Organización Internacional del Trabajo (2020) en conjunto con el de la Secretaría Ejecutiva de la Comisión Económica para América Latina y el Caribe (2020), que data del presente año en curso y que señala que serán tres los aspectos en los cuales existirá una mayor afectación laboral a raíz del desarrollo de la pandemia por COVID19. Para ello, se presenta el siguiente recuadro informativo a estos fines pertinentes: 


\section{Tabla 1.}

EFECTOS EN EL EMPLEO A RAIZ DE LA COVID-19 SEGÚN LA OIT (2020) Y LA CEPAL (2020)

\begin{tabular}{|l|l|}
\hline $\begin{array}{l}\text { CANTIDAD EN EL } \\
\text { EMPLO }\end{array}$ & $\begin{array}{l}\text { Disminuirá considerablemente: 5,3 millones en su caso } \\
\text { más favorable y } 24,7 \text { millones de desempleados en el } \\
\text { caso menos favorable }\end{array}$ \\
\hline CALIDAD DEL EMPLEO & $\begin{array}{l}\text { Puede disminuir considerablemente por las condiciones } \\
\text { económicas y a su vez por motivo de las faltas de control } \\
\text { en las empresas de trabajo presencial a raíz de la } \\
\text { pandemia así como por la implementación del } \\
\text { teletrabajo en otras cuestión que incide en los } \\
\text { trabajadores que tienen pocas o nulas condiciones de } \\
\text { internet en sus hogares. }\end{array}$ \\
\hline EFECTOS EN GRUPOS & $\begin{array}{l}\text { Será mucho más grave para estos grupos vulnerables. } \\
\text { Según la CEPAL a raíz de la pandemia coviD-19 el } \\
70,4 \% \text { de las trabajadoras domésticas están afectadas } \\
\text { por las medidas de la cuarentena, por disminución de la } \\
\text { actividad económica, desempleo reducción de las horas } \\
\text { o pérdidas de salarios. }\end{array}$ \\
\hline
\end{tabular}

Elaborado por: Bermeo y Naranjo Fuente: OIT (2020) y CEPAL (2020).

Al respecto, es importante mencionar que todas estas cifras a pesar de ser estimaciones podrían llegar a ser considerablemente mucho más altas que las esperadas y también mucho mayores a la crisis mundial del año 2008.

Es por esto que la pandemia ha agudizado las desigualdades y las crisis sistémicas previas y tiene impactos significativos en las trabajadoras del hogar en particular. También es deber de los estados reconocer y proteger sus derechos para que nadie se quede atrás. Acerca de la desigualdad se puede establecer que va de la mano con otros factores como el de la calidad del trabajo que afecta especialmente a las trabajadoras domésticas y de cuidados. Es por ello que se tienen que abrir espacios de diálogo y considerar las propuestas de los sindicatos como parte de las respuestas 
a la crisis y estrategias de recuperación socioeconómica que promueven los gobiernos y las instituciones financieras regionales e internacionales.

De acuerdo con el plan de respuesta humanitaria COVIG19 Ecuador en conjunto con las Naciones Unidas indica lo siguiente:

La pandemia afecta las diferentes esferas de la sociedad, extendiendo el sufrimiento de las personas y paralizando la economía. Las mujeres, niños, niñas y adolescentes, adultos mayores, personas en situación de calle, pueblos indígenas, afrodescendientes y montubios, personas refugiadas y migrantes residentes en el país y en tránsito, personas que viven en pobreza, extrema pobreza o trabajan en el sector informal, entre otros, son los grupos particularmente afectados.

Las mujeres están participando mayoritariamente en la respuesta a la enfermedad, incluso como trabajadoras de atención médica de primera línea, cuidadoras en el hogar y líderes comunitarias. La experiencia de otros brotes de enfermedades muestra que esta carga de atención también aumenta su riesgo de infección. A nivel mundial, las mujeres representan el $70 \%$ de los trabajadores del sector social y de salud y realizan tres veces más trabajo no remunerado que los hombres. Cuando los sistemas de salud están sobrecargados, se pone una mayor carga en la atención en el hogar y esa carga recae principalmente en las mujeres.

Por todo lo planteado se presenta el objetivo principal de esta investigación es analizar las proyecciones laborales para las trabajadoras domésticas en el contexto del COVID-19. En Ecuador y la vulneración de su trabajo.

\section{METODOLOGÍA}

La investigación se desarrolló desde la perspectiva cuantitativa, de tipo documental, ya que se puntualiza únicamente en la compilación de información de diversas fuentes. El diseño se circunscribe al bibliográfico, fundamentado en la revisión sistemática, rigurosa y profunda de material documental. Dicho diseño utiliza procedimientos lógicos y mentales tales como: análisis, síntesis, deducción e inducción, entre otros aspectos. (Palella Stracuzzi y Martins Pestana 2012) Por todo lo indicado se realizó un análisis de contenido de normativas, leyes, informes, datos 
de organismo mundiales, en el que se muestra la condición del trabajo doméstico en el contexto del COVID-19.

\section{RESULTADO}

Una evaluación global de la legislación y de las prácticas relativas al trabajo doméstico en el mundo indica que este tipo de trabajo se encuentra "infravalorado, mal remunerado, desprotegido y mal reglamentado" a pesar de las contribuciones que realizan los trabajadores domésticos al cuidado y bienestar de millones de hogares (OIT, 2009). Para Lexartza \& otros (2016), el trabajo doméstico es una de las ocupaciones con mayores tasas de empleo informal. La OIT estima que alrededor de 18 millones de personas se dedican al trabajo doméstico, aproximadamente $7 \%$ de todos los ocupados en la región. En 2013, la tasa de informalidad para las trabajadoras domésticas en América Latina era de 77,5\%. Es decir, casi 8 de cada 10 trabajadoras domésticas tenían un trabajo informal. Sin embargo, esta tasa se ha reducido en 2,6 puntos porcentuales entre 2009 y 2013, tal como ha sucedido en otros sectores.

\section{De acuerdo con cifras del Consejo Nacional para la Igualdad de Género}

La Encuesta del Uso del Tiempo (INEC 2012) refleja que el tiempo total de trabajo de las mujeres a la semana, es de 17:42 horas más que los hombres y el tiempo de trabajo doméstico y de cuidados no remunerado destinado por las mujeres es 3,5 veces más que el de los hombres, trabajo que representó en 2017 el 19,1\% de aporte al Producto Interno Bruto (PIB), es decir 19.872.978 USD.

Los roles de género tradicionalmente asignados a hombres y mujeres sobrevienen en la feminización de las profesiones relacionadas con los cuidados, son las mujeres quienes están mayoritariamente en la primera línea de respuesta a la crisis sanitaria y expuestas a un mayor riesgo de infección por las profesiones y tareas que ejercen como trabajadoras y profesionales sanitarias, voluntarias comunitarias y cuidadoras. Según el Registro Estadístico de Recursos y Actividades de Salud (INEC 2018) en el país hay 39.908 médicos/as y 24.751 enfermeras/os y 17.350 auxiliares de 
enfermería, quienes laboran en diferentes hospitales y centros médicos con distintos niveles de responsabilidad.

En contextos de emergencia la violencia contra las mujeres basada en género, particularmente la violencia intrafamiliar aumenta debido a las tensiones y conflictos en el hogar. Si bien la medida de aislamiento domiciliario es necesaria para prevenir la propagación del COVID 19, muchas mujeres se ven forzadas a permanecer con sus agresores en el hogar, lo cual pone en riesgo sus vidas. EI INEC reporta que el 65\% de las mujeres en Ecuador ha vivido violencia basada en género, el $45 \%$ perpetrada por su pareja. Las personas sobrevivientes de violencia pueden enfrentar obstáculos adicionales para huir de situaciones violentas y para acceder a medidas de protección o servicios que puedan salvar sus vidas, debido a factores como las restricciones de la circulación o la cuarentena. El hecho de que el conjunto de la población, en circunstancias como la de esta emergencia sanitaria, pueda ser considerada "vulnerable" nos muestra la importancia de resolverla necesidad social del cuidado, que no implique hogares sobrecargados, asumiendo los cuidados y una desigualdad de género que afecta mayormente a las mujeres, por la división sexual del trabajo, tanto en el ámbito remunerado como en la distribución de tareas y actividades en el interior de los hogares.

Durante la crisis sanitaria y económica resultado de la pandemia del COVID, la OIT viene apoyando campañas de sensibilización para mantener los ingresos de las trabajadoras domésticas y promover medidas de seguridad y salud para prevenir el contagio tanto en sus propios hogares como el de sus empleadores y en el tránsito al trabajo.

A corto plazo la crisis está incidiendo en la pérdida de empleo para las trabajadoras domésticas, a un futuro esto se convertirá en una falta total de fuentes de trabajo para estas mujeres, además de que las remuneraciones seguirán en caída y si no se implementan políticas para proteger la estabilidad económica de las trabajadoras, se pondrá en riesgo inclusive su entorno familiar. Además de que se podría establecer que la consideración de las mujeres como un grupo vulnerable viene a razonarse en el hecho de que suelen formar parte de colectivos de empleo más desprotegidos como 
el empleo temporal o en lo que ella denomina ocupaciones elementales, como las domesticas.

Es de conocimiento general que las trabajadoras domésticas se encargan de todo tipo de actividades dentro del hogar de sus empleadores, esto también incluye la administración de medicamentos cuando ellos se encuentran delicados de salud.

\section{Recomendaciones para garantizar los derechos y abordar los impactos del COVID-19 en las trabajadoras domésticas}

Impulsar medidas para garantizar la retención de empleos en el trabajo doméstico. Ampliar y promover las prestaciones por desempleo para trabajadoras domésticas. Garantizar transferencias monetarias o subsidios sociales de emergencia.

Elaborar protocolos de salud y seguridad que atiendan las especificidades del sector de trabajo doméstico remunerado.

Garantizar la cobertura en salud para todas las trabajadoras domésticas.

Asegurar que los servicios de cuidados sean considerados prioritarios.

Fomentar la formalización del trabajo doméstico.

Promover sistemas de protección social integrales.

Fomentar el diálogo social.

Promover la formación digital y la inclusión financiera de las trabajadoras domésticas. Asegurar el acceso a la información y a la asesoría legal para las trabajadoras domésticas.

Impulsar campañas de sensibilización sobre los derechos de las trabajadoras domésticas.

Impulsar la ratificación e implementación de los Convenios 189 y 190 de la OIT. 


\section{DISCUSIÓN}

Con todo esto puede denotar que las proyecciones y las hipótesis serias acerca del futuro de las mujeres y su relación con el mundo laboral, no son para nada las más alentadoras. Dado que, la crisis económica que ha dejado esta pandemia no avizora que las remuneraciones para las trabajadoras domésticas puedan aumentar, es más, podría verse una rebaja bastante notable en cuanto a sus sueldos, en el mejor de los casos. La OIT (2020) ante la actual coyuntura, organizaciones de trabajadoras remuneradas del hogar han denunciado que trabajadoras "cama adentro" tienen condiciones de abuso extremo impuestas por sus empleadores: se les incrementa la carga de trabajo, se les alarga la jornada laboral (de por sí ya extenuante), y no se les ofrece el equipo de protección personal indispensable para evitar ser contagiadas ni los implementos que garanticen la higiene en la vivienda. (p.1)

Asimismo, se espera que la mayoría de las trabajadoras del hogar terminen por perder sus empleos ya que sus empleadores prescindirán de sus servicios por el tiempo que ahora pasan las familias en casa y se ocupan de sus propias necesidades. Por otro lado, aquellas trabajadoras domésticas que continúen con un trabajo y se mantenga regular su remuneración, seguramente tendrán que cumplir con más horas laborables e inclusive prestar servicios puertas adentro de los hogares.

Desde otro punto de vista como lo es el de la salud, los empleadores deben hacer el compromiso de brindar todas las seguridades y protocolos necesarios para prevenir el contagio y propagación del COVID-19, aunque es un tema bastante complicado de llevar a la realidad ya que ni las grandes empresas en muchos de los casos ofrecen las óptimas condiciones para sus trabajadores, pero en la medida de lo posible se debe implementar esta protección voluntariamente por sus empleadores, ya que las empleadas domésticas al no poseer una representación sindical que vele por su bienestar, deben buscar las mejores alternativas para proteger su salud e integridad. 


\section{CONCLUSIONES}

Se debe considerar que las trabajadoras domésticas estuvieron entre los primeros sectores afectados por la emergencia sanitaria a causa del coronavirus. Esto porque no han podido asistir a realizar sus labores, o en otros casos, los empleadores no les han permitido ingresar a sus lugares de trabajo por el temor de que se encuentren contagiadas del COVID-19 y esto se ha visibilizado en la crisis de este grupo laboral. En tal virtud, las consecuencias de este detenimiento de las actividades van a calar muy hondo y depende tanto de empleadas como empleadores contribuir para el resurgimiento de este sector que es el medio de vida para varias familias.

Por otro lado, se debe continuar con la tarea de presionar a las autoridades correspondientes para que tomen cartas en el asunto y plateen alternativas con el fin proteger y tutelar los derechos de las trabajadoras domésticas, ya que la realidad que vendrá después de la pandemia seguramente será aún más complicada, por tal motivo, este sector de la sociedad debe contar con las garantías necesarias para continuar con la prestación de sus servicios y a la vez tener una estabilidad tanto laboral como económica.

\section{FINANCIAMIENTO}

No monetario.

\section{AGRADECIMIENTO}

A la Universidad Regional Autónoma de Los Andes; por motivar el desarrollo de la Investigación. 


\section{REFERENCIAS CONSULTADAS}

Asamblea Nacional Constituyente de la República del Ecuador, (2008). Constitución de la República del Ecuador. Montecristi. Registro Oficial 449 de 20-oct-2008. Recuperado de https://n9.cl/sia

Caamaño-Rojo, E. (2010). Mujer y trabajo: origen y ocaso del modelo del padre proveedor y la madre cuidadora. [Woman and Work: Source and Decline of the Provider Father and the Caregiving Mother Model]. Revista de Derecho de la Pontificia Universidad Católica de Valparaíso, 6,179-209. http://dx.doi.org/10.4067/S0718-68512010000100005

Consejo Nacional para la Igualdad de Género (2020). Los riesgos a los que se enfrentan las mujeres durante la amenaza sanitaria por el COVID-19 en Ecuador. [The risks women face during coVID-19 health threat in Ecuador]. Recuperado de: https://n9.cl/v0ajl

Lexartza, L., Chaves, María José \& Carcedo, A. /2016). Políticas de formalización del trabajo doméstico remunerado en América Latina y el Caribe. [ Policies for the formalization of paid domestic work in Latin America and the Caribbean.] Lima, OIT, Oficina Regional para América Latina y el Caribe, FORLAC, 52 p. Recuperado de: https://n9.cl/e88nn

Naciones Unidas (2020). Plan de Respuesta humanitaria COVID-19 Ecuador. [COVID-19 Ecuador Humanitarian Response Plan]. Recuperado de: https://n9.cl/m7ak

OIT (2009): Trabajo decente para los trabajadores domésticos, Informe IV (1), Conferencia Internacional de Trabajo, 99.a reunión, 2010.

Organización Internacional del Trabajo (2020). El trabajo del hogar en tiempos de COVID-19. [Home work in COVID-19 times]. Recuperado de: https://n9.cl/3w9va

ONU Mujeres-OIT-CEPAL (2020). Trabajadoras remuneradas del hogar en América Latina y el Caribe frente a la Crisis del COVID-19. [Paid domestic workers in Latin America and the Caribbean in the face of the COVID-19 Crisis]. Recuperado de: https://n9.cl/5o26u

Palella Stracuzzi, S. \& Martins Pestana, F. (2012). Metodología de la investigación cuantitativa. [Quantitative research methodology]. Fondo editorial de la Universidad Pedagógica Libertador. Caracas, Venezuela. 
Iustitia Socialis. Revista Arbitrada de Ciencias Jurídicas.

Año V. Vol. V. №3. Edición Especial. 2020-III:

Universidad Regional Autónoma de los Andes

Hecho el depósito de Ley: FA2016000064

ISSN: 2542-3371

FUNDACIÓN KOINONIA (F.K). Santa Ana de Coro, Venezuela

Mercedes Carolina Navarro Cejas; Lola Ximena Cangas-Oña; Jhoanna Nicole Bermeo-Alvarado Cristian Patricio Naranjo-Moreno

Sánchez, Jorge E., José Luis Sánchez-Espinosa, José L., Jorge Hernando SánchezEspinosa, Jorge H, \& Sánchez-Espinosa, V. (2019). La igualdad de la mujer y el derecho al trabajo en Ecuador. [Women's equality and the right to work in Ecuador]. KOINONIA. 6(8), 39-63. http://dx.doi.org/10.35381/racji.v4i7.354

2020 por los autores. Este artículo es de acceso abierto y distribuido según los términos y condiciones de la licencia Creative Commons Atribución-NoComercial-Compartirlgual 4.0 Internacional (CC BY-NC-SA 4.0) (https://creativecommons.org/licenses/by-nc-sa/4.0/). 